URL www.una.ac.cr/mhsalud

\title{
APRENDIZAJE DE LAS MATEMÁTICAS POR MEDIO DEL MOVIMIENTO: UNA ALTERNATIVA MÁS DE LA EDUCACIÓN FÍSICA
}

\author{
*Serrano Madrigal Ariana, *Azofeifa Lizano Ana $y^{1 \text { y }}{ }^{2}$ Araya Vargas Gerardo \\ * Preescolar Bilingüe Santa Cecilia y ${ }^{1}$ Escuela Ciencias del Deporte, Universidad Nacional y \\ ${ }^{2}$ Escuela de Educación Física y Deportes, Universidad de Costa Rica, Costa Rica \\ E-mail: anairaonarres@gmail.com; anaazofeifa@gmail.com
}

RESUMEN

\begin{abstract}
El propósito de este estudio fue determinar los efectos en el nivel cognitivo, en dos grupos de estudiantes de preparatoria, después de aplicar actividades físico recreativas, relacionadas en un grupo con contenidos de matemáticas y en el otro grupo con juegos recreativos. Participaron 27 sujetos (13 niñas y 14 niños), con edades entre 5 y medio y 6 y medio años. El instrumento que se utilizó fue un cuestionario sobre elementos fundamentales de las matemáticas, basados en los temarios del Ministerio de Educación Pública de Costa Rica, como lo fueron, geometría, operaciones básicas con elementos concretos y conocimiento del reloj. Una vez confeccionado el instrumento, se realizó un plan de actividades físico recreativas relacionadas a las matemáticas, el cual fue desarrollado durante un mes y medio con el grupo experimental (preparatoria B), mientras el otro grupo realizaba juegos recreativos. Para el análisis de los datos se utilizó tanto estadística descriptiva como inferencial. Se encontraron efectos significativos y positivos del programa de actividades físico recreativas sobre el rendimiento de los y las estudiantes en 10 de los 12 items que fueron aplicados para valorar el dominio de conceptos básicos de las matemáticas. Se puede concluir que al utilizar la educación física como un instrumento más para el aprendizaje de otras disciplinas, representa una excelente alternativa para los docentes de preescolar que buscan satisfacer las necesidades de aprendizaje de los niños y niñas que pronto irán a la escuela. El movimiento por medio de experiencias guiadas y planeadas, es parte indispensable en la vida de los niños y niñas, por lo tanto, el aprendizaje de materias académicas debe adaptarse a la necesidad que ellos y ellas tiene de explorar y conocer su entorno.
\end{abstract}

PALABRAS CLAVES: educación física, matemáticas, actividades físico-recreativas.

\section{LEARNING MATH THROUGH MOVEMENT: PHYSICAL EDUCATION AS ANOTHER ALTERNATIVE}

\section{ABSTRACT}

The purpose of this study was to determine the cognitive effects of applying physical recreational activities to two groups of pre-school students, related to mathematics to one of the groups and recreational games to the other. A total of 27 subjects (13 girls and 14 boys) of 5 and a half and 6 and half years of age participated in the study. The instrument used was a questionnaire including basic math concepts such as geometry, basic operations with concrete elements, and how to read the clock, based on the topics established by the Costa Rican Ministry of Public Education. Once the instrument was developed, a plan of physical recreational activities related to math was prepared and applied to the experimental group (pre-school B) for one and a half months, while the other group played recreational games. Data was analyzed using descriptive and inferential statistics. Positive and significant effects were found in the physical recreational activity program regarding student performance in 10 of the 12 items that were applied to assess mastery of basic math concepts. In conclusion, using physical education as another instrument to teach other disciplines represents an excellent alternative for pre-school teachers that try to satisfy the learning needs of children that will soon be attending school. Using movement as part of guided and planned activities plays an indispensable role in children's lives; therefore, learning academic subjects should be adapted to their needs to explore and know their environment.

KEY WORDS: physical education, mathematics, physical-recreational activities. 


\section{MHSalud}

URL www.una.ac.cr/mhsalud

\section{INTRODUCCIÓN}

El niño o la niña puede mediante el movimiento, aprender y lograr una mejor capacidad de rendimiento en todas las áreas de la vida. Es decir en lo social, en lo intelectual, en lo propiamente motor o del movimiento y en lo emocional (Bolaños, 1991).

Uno de los primeros investigadores que estudió y relacionó el aspecto cognitivo con el psicomotor, fue Jean Piaget; sus estudios se orientaron más al campo de desarrollo intelectual del niño(a) en la escuela primaria. Piaget dio mucha importancia a la relación entre el movimiento y el intelecto, debido a que según su teoría, el(a) niño(a) abstrae la información que utiliza del movimiento (Rodríguez y Vega, 2004 y Woodburn, 1985).

Sanabria (1995), expresa que para lograr un desarrollo integral en los seres humanos, existen 3 niveles: el cognoscitivo, el afectivo y el psicomotor; siendo necesario contar con una amplia gama de experiencias de aprendizaje por medio de las actividades físicas que permitan la estimulación de los niveles antes mencionados.

Sin embargo, han existido investigadores que han incursionado en el tema del nivel cognitivo y el movimiento físico, es por ello que se mencionarán algunos de los estudios relacionados con este tema. Por ejemplo, Bolaños (1991) comenta que se ha demostrado que al relacionar los contenidos de una materia educativa con las de otra, se experimenta un mejoramiento en relación con el aprendizaje de cada una de ellas.

Existen varios estudios que han representado por medio de sus resultados, los beneficios de la actividad física y juegos recreativos a nivel cognitivo: Aguilar, 1990; Sanabria, 1995; Alfaro y Salazar, 2001; Díaz, 1996; Rodríguez y Vega, 2004; Tomporowsky y Ellis, 1986; Montes de Oca y Romero, 2003; Gondola, 1987; Zervas, 1990; McMorris y Graydon, 2000; Etnier, Salazar, Landers, Petruzzello, Han y Novell (1997), entre otros.

Serrano, Ramírez y Araya (en prensa) comprobaron que los niños y niñas que asisten semanalmente a una Escuela Deportiva Pedagógica obtuvieron diferencias significativas en cuanto a su nivel cognitivo (test de Raven) y de actividad física, en comparación a niños(as) que no son estimulados a través de actividades físicas sistemáticas. Al niño por naturaleza le gusta jugar. También hay que ser consciente que el mundo de los seres humanos está enteramente ligado al mundo de los objetos; donde el (la) niño(a) es un elemento más que interactúa con el resto de los mismos (Morales, 1985).

Bustos et al. (1999), expresan que la educación es como un conjunto de prácticas que utiliza un grupo social con el fin de favorecer el desarrollo individual de sus miembros, que asimilarán la experiencia colectiva culturalmente organizada y se convertirá a la vez en agentes de creación y cambio cultural.

No obstante, una educación intelectualizada impide y bloquea toda la manifestación espontánea y libre de la infancia; incluso, con frecuencia, los educadores convierten a los juegos infantiles en meros instrumentos de instrucción, por lo que pierden su legítimo lugar, como manifestación del ser pleno de alegría y completa libertad (Zapata, 1989). 


\section{MHSalud}

URL www.una.ac.cr/mhsalud

Es importante mencionar que existen conceptos de difícil comprensión para los niños y niñas, los que pueden ser entendidos por ellos de una mejor manera si se enseñan por medio de movimientos sencillos. Así, los niños(as) pequeños(as) pueden adquirir mediante el movimiento los conceptos: arriba, abajo, sobre, debajo, rápido, lento; los niños(as) mayores pueden adquirir conceptos geométricos mientras caminan formando una figura, etc. (Bolaños, 1991).

El movimiento, el juego y las actividades físicas y deportivas constituyen para la y el infante de edad escolar, procesos de aprendizaje que le permiten satisfacer sus profundas necesidades de actividad, de diversión y de exploración, además, ayuda a que se produzcan cambios de conducta en la personalidad del niño(a), como producto de un conocimiento más amplio de su cuerpo y de su ambiente (Bolaños, 1991; Decker, 1981).

Zapata (1989), expresa que es de gran utilidad el uso de los juegos de aprestamiento para la lectura, la escritura y las matemáticas, ya que tienen la finalidad de iniciar al niño(a) en las actividades que lo conducirán al aprendizaje de estas disciplinas en el primer y segundo grado; a la vez, que prepararlo para la adquisición de estas habilidades dentro de un marco afectivo.

En este contexto se debe considerar el movimiento corporal como un método o un medio más, incorporado directamente al aprendizaje de conceptos académicos, las que casi siempre el infante y los maestros llevan a cabo en forma pasiva y dentro del aula (Bolaños, 1991).

En la enseñanza de las matemáticas se debe tener presente que los conceptos básicos en que ésta materia se desarrolla, están presentes en la vida real, propias o al alcance de las experiencias del educando, tales como: poesías, canciones, juegos, láminas, cuentos y dramatizaciones (Morales, 1985; Rey, 2001).

Dado que el conocimiento matemático es una herramienta básica para la comprensión y el manejo de la realidad que vive el niño(a) y está presente en la vida diaria desde edades muy tempranas, podría decirse que las primeras experiencias de los(as) niños(as) con las matemáticas son a través del movimiento y los juegos.

El niño(a) a los seis años domina conceptos prearitméticos, clasifica objetos según criterios determinados (color, forma, tamaño, grosor), forma series de objetos, llega a conceptos de orden, serie, grupo, mediante comparación, clasificación y ordenación de objetos, cuenta con diversos modos, desplazando, agrupando, tocando, se familiariza con el reloj, cuenta de veinte a más números, posee relaciones asimétricas (ancho-angosto, grueso-delgado, pesado-liviano) (Gran Libro de la Maestra de Preescolar, 2003).

La educación física hace suyos los objetivos generales de la educación, como parte integrante de ella, con aportaciones muy específicas. Constituye un elemento irremplazable para la formación de la personalidad. Junto con la expresión oral, el lenguaje matemático, y la expresión escrita, el lenguaje corporal y las conductas motrices permiten al hombre expresarse, comunicarse, realizarse plenamente (Bolaños, 1991; Cagigal, 1979; Rey, 2001 y Trigo, 1992).

No obstante, con frecuencia la clase de educación física ha sido criticada por docentes y padres de familia, quienes afirman que en ella se pierde el tiempo, tiempo que el (la) niño(a) aprovecharía en su desarrollo mental e intelectual. Dicen esas personas que la escuela y el colegio deben preparar para el estudio y no para el deporte (Bolaños, 1991).

Sin embargo, la tendencia en algunas ocasiones de los educadores físicos es la integración de contenidos cognitivos en sus unidades didácticas, lo que facilita trabajarlos más a través de la práctica (Rey, 2001). 


\section{MHSalud}

URL www.una.ac.cr/mhsalud

Es por esto que el maestro no tiene porqué limitar la educación de motriz de sus alumnos a la sesión de educación física, si puede prolongarla a otros momentos de la jornada escolar y bajo la perspectiva de otras materias curriculares tal como se ha dicho anteriormente ya que la educación física también puede ofrecer numerosas aportaciones a otras áreas de la educación infantil.

No obstante, la idea no es ensalzar el valor de la educación física atribuyéndole efectos maravillosos en las diversas materias escolares, sino simplemente reflexionar acerca de las posibilidades de globalización relacionadas con la educación física. Por tales razones, se encuentra en los juegos recreativos, una opción para reforzar las necesidades académicas del niño(a) de manera activa, creativa y divertida. La actividad recreativa proporciona oportunidades para que cada niño y niña se desarrolle social, emocional, intelectual y físicamente (Céspedes, 1987; Villalobos, 2001; Bolaño y Thomas, 1994 y Zamora, 1988).

Es en el juego infantil donde se proporcionan todas las posibilidades de una vida feliz; solamente se debe tratar, que en el encuentro que tienen los y las niñas en este tiempo, se aproveche completamente estas posibilidades, reconociendo primeramente toda su amplitud; no obstante, tiene gran importancia instructiva y educativa en la vida de las personas (Moor, 1987; Rudik, 1986; Vaca, 1983; Lavega, 2000).

Huizinga (1954), expresó textualmente que: "el juego es una acción u ocupación libre, que se desarrolla dentro de unos límites temporales y espaciales determinados, según reglas absolutamente obligatorias, aunque libremente aceptadas, acción que tiene un fin en sí misma y va acompañada de un sentimiento de tensión y alegría y de la conciencia de ser de otro modo que en la vida corriente" pág. 43.

Según Rudik (1986), los juegos infantiles se caracterizan por las siguientes particularidades: la libre creación y la independencia del niño, esto no significa que en el juego no haya obligaciones y reglas a las que hay que subordinarse, sin embargo, la libre manifestación de la actividad empezada, continuada y terminada voluntariamente es una particularidad característica del juego.

El carácter vivo de la actividad, el juego nunca puede consistir en repeticiones de una actividad automatizada, por eso, sería erróneo reducir el juego a la ejecución mecánica y carente de interés, de operaciones de juego establecidos de una vez para siempre. La saturación emocional, la actividad del juego va siempre acompañada de un sentimiento de satisfacción que le es característico.

Lleixa (1993), expresa mediante el siguiente resumen la globalidad de la educación física en las edades de 3 a 8 años: 

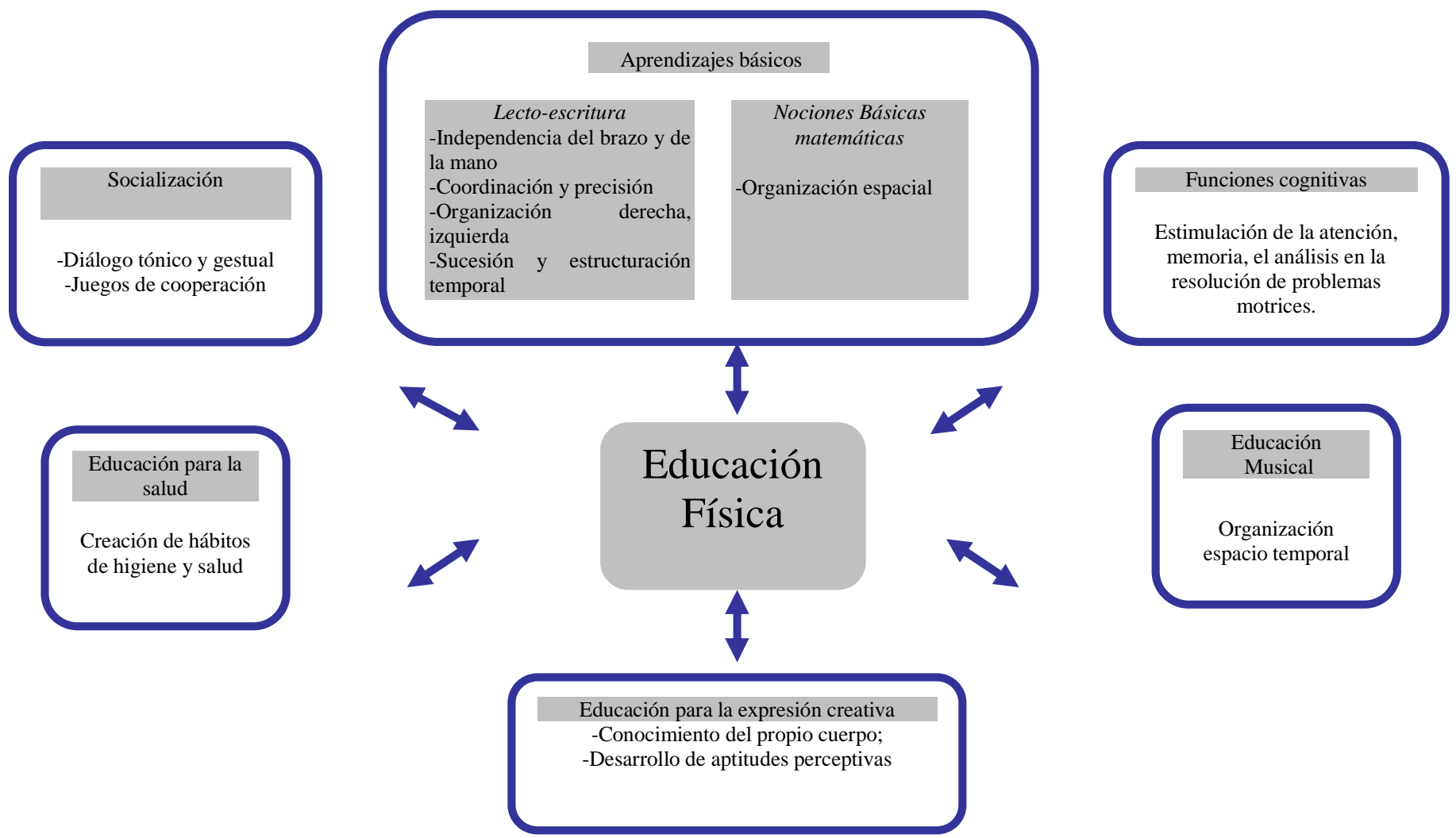

Educación para la salud

Creación de hábitos de higiene y salud

El propósito de esta investigación fue determinar los efectos a nivel cognitivo en dos grupos de estudiantes de preparatoria, después de aplicar actividades físico recreativas relacionadas con contenidos de matemáticas a uno de ellos y a los otros, juegos recreativos.

\section{METODOLOGÍA}

Sujetos: En este estudio participaron 27 estudiantes de los cuales 13 eran niñas y 14 niños del Preescolar Bilingüe Santa Cecilia, ubicado en Heredia, durante el curso lectivo 2004, con edades entre los cinco y medio y seis y medio años, que cursaban el nivel de preparatoria. Para efectos de la investigación, se dividió la población en dos grupos, uno control con 14 sujetos ( 7 niños y 7 niñas) y el experimental con 13 sujetos ( 7 niños y 6 niñas).

Instrumentos y materiales: El instrumento que se utilizó fue un cuestionario realizado por las investigadoras, el cual fue avalado por expertos, sobre elementos fundamentales de las matemáticas, basados en los temarios del Ministerio de Educación Pública de Costa Rica. Estos temas fueron: geometría, operaciones básicas con elementos concretos y conocimiento del reloj. Consta de 4 ítems por tema, para un total de 12 ítemes. En contraparte, se confeccionó el plan de actividades físico recreativas relacionadas a las matemáticas, el cual tuvo una duración de mes y medio.

El estudiante realiza las siguientes actividades según los 12 ítemes que se especifican a

continuación: Revista MHSalud® (ISSN: 1659-097X) Vol. 5. No. 2. Diciembre, 2008. 
URL www.una.ac.cr/mhsalud

Tabla 1. Ítems evaluados en el instrumento

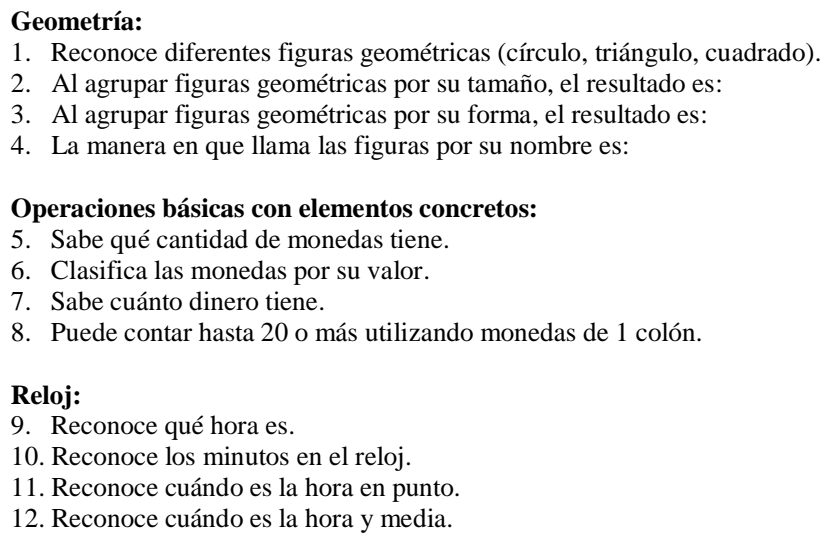

Procedimiento: Para realizar esta investigación en primera instancia se tuvo que confeccionar el cuestionario que se aplicaría a los estudiantes. Este cuestionario fue avalado por expertos. Posteriormente fue aplicado como pretest a 27 niños y niñas de las preparatorias A y B de la institución. Paralelamente, se confeccionó un plan de actividades físico recreativas relacionadas a las matemáticas, el cual sería desarrollado durante un mes y medio en las lecciones previstas para la natación con el grupo experimental (preparatoria B). De las dos lecciones semanales una se destinó para educación física y la otra para desarrollar el proyecto a la cual se le llamó "educación por medio del movimiento". Al concluir las 6 semanas, se aplicó nuevamente la prueba para determinar si el tratamiento trajo algún cambio en el aprendizaje de las matemáticas. Finalmente se realizó la tabulación de datos, análisis estadístico y discusión de los resultados.

Análisis Estadístico: Para el análisis de los datos se trabajó como estadística descriptiva, promedios y desviaciones estándar. Como estadística inferencial se aplicó análisis de varianza de 3 vías, mixto con medidas repetidas en un solo componente.

\section{RESULTADOS}

A continuación se muestran los principales resultados que mostró la investigación:

Tabla 2. Resumen de promedios y desviaciones estándar de los puntajes obtenidos en el ítem 1

\begin{tabular}{|c|c|c|c|c|c|}
\hline & Grupo & Sexo & Promedio & Desviación estándar & $\mathbf{n}$ \\
\hline \multirow[t]{9}{*}{ Pre } & grupo control & mujer & 4,1429 & $\pm 1,0690$ & 7 \\
\hline & & hombre & 3,8571 & $\pm 1,0690$ & 7 \\
\hline & & Total & 4,0000 & $\pm 1,0377$ & 14 \\
\hline & grupo experimental & mujer & 2,8333 & $\pm 1,1690$ & 6 \\
\hline & & hombre & 4,0000 & $\pm 1,0000$ & 7 \\
\hline & & Total & 3,4615 & $\pm 1,1983$ & 13 \\
\hline & Total & mujer & 3,5385 & $\pm 1,2659$ & 13 \\
\hline & & hombre & 3,9286 & $\pm 0,9972$ & 14 \\
\hline & & Total & 3,7407 & $\pm 1,1298$ & 27 \\
\hline \multirow[t]{9}{*}{ Post } & grupo control & mujer & 4,2857 & $\pm 1,1127$ & 7 \\
\hline & & hombre & 4,0000 & $\pm 1,7321$ & 7 \\
\hline & & Total & 4,1429 & $\pm 1,4064$ & 14 \\
\hline & grupo experimental & mujer & 4,1667 & $\pm 0,7528$ & 6 \\
\hline & & hombre & 4,4286 & $\pm 1,1339$ & 7 \\
\hline & & Total & 4,3077 & $\pm 0,9473$ & 13 \\
\hline & Total & mujer & 4,2308 & $\pm 0,9268$ & 13 \\
\hline & & hombre & 4,2143 & $\pm 1,4239$ & 14 \\
\hline & & Total & 4,2222 & $\pm 1,1875$ & 27 \\
\hline
\end{tabular}




\section{MHSalud}

URL www.una.ac.cr/mhsalud

Tabla 3. Resumen de análisis de varianza de los puntajes obtenidos en el ítem 1 según sexo, grupo y medición

\begin{tabular}{cccc}
\hline Fuente & F & Sig. & Eta $^{2}$ \\
\hline MEDICION & 7,803 & $0,010 *$ & 0,253 \\
MEDICION * GRUPO & 4,056 & 0,056 & 0,150 \\
MEDICION * SEXO & 1,523 & 0,230 & 0,062 \\
MEDICION * GRUPO * SEXO & 1,523 & 0,230 & 0,062 \\
GRUPO & 0,272 & 0,607 & 0,012 \\
SEXO & 0,272 & 0,607 & 0,012 \\
GRUPO * SEXO & 1,482 & 0,236 & 0,061 \\
$* \mathrm{p}<0,05$ & & &
\end{tabular}

Tabla 4. Resumen de promedios y desviaciones estándar de los puntajes obtenidos en el ítem 2

\begin{tabular}{|c|c|c|c|c|c|}
\hline \multirow{2}{*}{ Pre } & \multirow{2}{*}{$\begin{array}{c}\text { Grupo } \\
\text { grupo control }\end{array}$} & \multirow{2}{*}{$\begin{array}{c}\text { Sexo } \\
\text { mujer }\end{array}$} & \multicolumn{2}{|c|}{ Promedio Desviación estándar } & \multirow{2}{*}{$\begin{array}{c}\mathbf{n} \\
7\end{array}$} \\
\hline & & & 3,8571 & $\pm 1,4639$ & \\
\hline & & hombre & 3,7143 & $\pm 1,3801$ & 7 \\
\hline & & Total & 3,7857 & $\pm 1,3688$ & 14 \\
\hline & grupo experimental & mujer & 3,1667 & $\pm 1,3292$ & 6 \\
\hline & & hombre & 3,2857 & $\pm 0,9512$ & 7 \\
\hline & & Total & 3,2308 & $\pm 1,0919$ & 13 \\
\hline & Total & mujer & 3,5385 & $\pm 1,3914$ & 13 \\
\hline & & hombre & 3,5000 & $\pm 1,1602$ & 14 \\
\hline & & Total & 3,5185 & $\pm 1,2518$ & 27 \\
\hline \multirow[t]{9}{*}{ Post } & grupo control & mujer & 4,0000 & $\pm 1,5275$ & 7 \\
\hline & & hombre & 3,7143 & $\pm 1,3801$ & 7 \\
\hline & & Total & 3,8571 & $\pm 1,4064$ & 14 \\
\hline & grupo experimental & mujer & 3,8333 & $\pm 1,6021$ & 6 \\
\hline & & hombre & 4,7143 & $\pm 0,4880$ & 7 \\
\hline & & Total & 4,3077 & $\pm 1,1821$ & 13 \\
\hline & Total & mujer & 3,9231 & $\pm 1,4979$ & 13 \\
\hline & & hombre & 4,2143 & $\pm 1,1217$ & 14 \\
\hline & & Total & 4,0741 & $\pm 1,2987$ & 27 \\
\hline
\end{tabular}

Tabla 5. Resumen de análisis de varianza de los puntajes obtenidos en el ítem 2 según sexo, grupo y medición

\begin{tabular}{cccc}
\hline Fuente & F & Sig. & Eta $^{2}$ \\
\hline MEDICION & 13,920 & $0,001^{* *}$ & 0,377 \\
MEDICION * GRUPO & 10,593 & $0,003 * *$ & 0,315 \\
MEDICION * SEXO & 1,065 & 0,313 & 0,044 \\
MEDICION * GRUPO * SEXO & 2,275 & 0,145 & 0,090 \\
GRUPO & 0,022 & 0,883 & 0,001 \\
SEXO & 0,088 & 0,769 & 0,004 \\
GRUPO * SEXO & 0,553 & 0,465 & 0,023
\end{tabular}

$\bar{*} \mathrm{p}<0,01$

Revista MHSalud® (ISSN: 1659-097X) Vol. 5. No. 2. Diciembre, 2008. 


\section{MHSalud}

URL www.una.ac.cr/mhsalud

Tabla 6. Resumen de promedios y desviaciones estándar de los puntajes obtenidos en el ítem 3

\begin{tabular}{|c|c|c|c|c|c|}
\hline & Grupo & Sexo & Promedio & Desviación estándar & $\mathbf{n}$ \\
\hline \multirow[t]{9}{*}{ Pre } & grupo control & mujer & 4,5714 & $\pm 0,5345$ & 7 \\
\hline & & hombre & 4,7143 & $\pm 0,4880$ & 7 \\
\hline & & Total & 4,6429 & $\pm 0,4972$ & 14 \\
\hline & grupo experimental & mujer & 4,0000 & $\pm 0,6325$ & 6 \\
\hline & & hombre & 3,8571 & $\pm 1,0690$ & 7 \\
\hline & & Total & 3,9231 & $\pm 0,8623$ & 13 \\
\hline & Total & mujer & 4,3077 & $\pm 0,6304$ & 13 \\
\hline & & hombre & 4,2857 & $\pm 0,9139$ & 14 \\
\hline & & Total & 4,2963 & $\pm 0,7753$ & 27 \\
\hline \multirow[t]{9}{*}{ Post } & grupo control & mujer & 4,5714 & $\pm 0,7868$ & 7 \\
\hline & & hombre & 4,4286 & $\pm 1,1339$ & 7 \\
\hline & & Total & 4,5000 & $\pm 0,9405$ & 14 \\
\hline & grupo experimental & mujer & 4,5000 & $\pm 0,5477$ & 6 \\
\hline & & hombre & 4,7143 & $\pm 0,4880$ & 7 \\
\hline & & Total & 4,6154 & $\pm 0,5064$ & 13 \\
\hline & Total & mujer & 4,5385 & $\pm 0,6602$ & 13 \\
\hline & & hombre & 4,5714 & $\pm 0,8516$ & 14 \\
\hline & & Total & 4,5556 & $\pm 0,7511$ & 27 \\
\hline
\end{tabular}

Tabla 7. Resumen de análisis de varianza de los puntajes obtenidos en el ítem 3 según sexo, grupo y medición

\begin{tabular}{cccc}
\hline Fuente & F & Sig. & Eta $^{2}$ \\
\hline MEDICION & 1,865 & 0,185 & 0,075 \\
MEDICION * GRUPO & 4,385 & $0,047^{*}$ & 0,160 \\
MEDICION * SEXO & 0,008 & 0,928 & 0,000 \\
MEDICION * GRUPO * SEXO & 0,671 & 0,421 & 0,028 \\
GRUPO & 1,969 & 0,174 & 0,079 \\
SEXO & 0,007 & 0,935 & 0,000 \\
GRUPO * SEXO & 0,007 & 0,935 & 0,000 \\
\hline
\end{tabular}

$* \overline{\mathrm{p}}<0,05$

Tabla 8. Resumen de promedios y desviaciones estándar de los puntajes obtenidos en el ítem 4

\begin{tabular}{|c|c|c|c|c|c|}
\hline & Grupo & Sexo & Promedio & Desviación estándar & $\mathbf{n}$ \\
\hline \multirow[t]{9}{*}{ Pre } & grupo control & mujer & 3,7143 & $\pm 0,9512$ & 7 \\
\hline & & hombre & 3,8571 & $\pm 0,8997$ & 7 \\
\hline & & Total & 3,7857 & $\pm 0,8926$ & 14 \\
\hline & grupo experimental & mujer & 3,6667 & $\pm 1,2111$ & 6 \\
\hline & & hombre & 3,8571 & $\pm 1,0690$ & 7 \\
\hline & & Total & 3,7692 & $\pm 1,0919$ & 13 \\
\hline & Total & mujer & 3,6923 & $\pm 1,0316$ & 13 \\
\hline & & hombre & 3,8571 & $\pm 0,9493$ & 14 \\
\hline & & Total & 3,7778 & $\pm 0,9740$ & 27 \\
\hline \multirow[t]{9}{*}{ Post } & grupo control & mujer & 3,7143 & $\pm 1,2536$ & 7 \\
\hline & & hombre & 4,4286 & $\pm 0,9759$ & 7 \\
\hline & & Total & 4,0714 & $\pm 1,1411$ & 14 \\
\hline & grupo experimental & mujer & 4,3333 & $\pm 1,2111$ & 6 \\
\hline & & hombre & 4,7143 & $\pm 0,4880$ & 7 \\
\hline & & Total & 4,5385 & $\pm 0,8771$ & 13 \\
\hline & Total & mujer & 4,0000 & $\pm 1,2247$ & 13 \\
\hline & & hombre & 4,5714 & $\pm 0,7559$ & 14 \\
\hline & & Total & 4,2963 & $\pm 1,0309$ & 27 \\
\hline
\end{tabular}

Revista MHSalud® (ISSN: 1659-097X) Vol. 5. No. 2. Diciembre, 2008. 


\section{MHSalud}

URL www.una.ac.cr/mhsalud

Tabla 9. Resumen de análisis de varianza de los puntajes obtenidos en el ítem 4 según sexo, grupo y medición

\begin{tabular}{cccc} 
Fuente & F & Sig. & Eta $^{2}$ \\
\hline MEDICION & 8,522 & $0,008^{* *}$ & 0,270 \\
MEDICION * GRUPO & 1,761 & 0,198 & 0,071 \\
MEDICION * SEXO & 1,127 & 0,299 & 0,047 \\
MEDICION * GRUPO * SEXO & 0,282 & 0,601 & 0,012 \\
GRUPO & 0,369 & 0,549 & 0,016 \\
SEXO & 1,026 & 0,322 & 0,043 \\
GRUPO * SEXO & 0,041 & 0,841 & 0,002
\end{tabular}

$* * \mathrm{p}<0,01$

Tabla 10. Resumen de promedios y desviaciones estándar de los puntajes obtenidos en el ítem 5

\begin{tabular}{|c|c|c|c|c|c|}
\hline & Grupo & Sexo & Promedio & Desviación estándar & $\mathbf{n}$ \\
\hline \multirow[t]{9}{*}{ Pre } & grupo control & mujer & 3,0000 & $\pm 0,0000$ & 7 \\
\hline & & hombre & 3,0000 & $\pm 0,0000$ & 7 \\
\hline & & Total & 3,0000 & $\pm 0,0000$ & 14 \\
\hline & grupo experimental & mujer & 2,6667 & $\pm 0,8165$ & 6 \\
\hline & & hombre & 2,2857 & $\pm 0,7559$ & 7 \\
\hline & & Total & 2,4615 & $\pm 0,7763$ & 13 \\
\hline & Total & mujer & 2,8462 & $\pm 0,5547$ & 13 \\
\hline & & hombre & 2,6429 & $\pm 0,6333$ & 14 \\
\hline & & Total & 2,7407 & $\pm 0,5944$ & 27 \\
\hline \multirow[t]{9}{*}{ Post } & grupo control & mujer & 2,5714 & $\pm 0,7868$ & 7 \\
\hline & & hombre & 2,8571 & $\pm 0,3780$ & 7 \\
\hline & & Total & 2,7143 & $\pm 0,6112$ & 14 \\
\hline & grupo experimental & mujer & 3,0000 & $\pm 0,0000$ & 6 \\
\hline & & hombre & 2,8571 & $\pm 1,3452$ & 7 \\
\hline & & Total & 2,9231 & $\pm 0,9541$ & 13 \\
\hline & Total & mujer & 2,7692 & $\pm 0,5991$ & 13 \\
\hline & & hombre & 2,8571 & $\pm 0,9493$ & 14 \\
\hline & & Total & 2,8148 & $\pm 0,7863$ & 27 \\
\hline
\end{tabular}

Tabla 11. Resumen de análisis de varianza de los puntajes obtenidos en el ítem 5 según sexo, grupo y medición

\begin{tabular}{cccc}
\hline Fuente & F & Sig. & Eta $^{2}$ \\
\hline MEDICION & 0,315 & 0,580 & 0,014 \\
MEDICION * GRUPO & 6,183 & $0,021^{*}$ & 0,212 \\
MEDICION * SEXO & 0,778 & 0,387 & 0,033 \\
MEDICION * GRUPO * SEXO & 0,006 & 0,937 & 0,000 \\
GRUPO & 0,481 & 0,495 & 0,020 \\
SEXO & 0,071 & 0,792 & 0,003 \\
GRUPO * SEXO & 0,823 & 0,374 & 0,035
\end{tabular}

$* \mathrm{p}<0,05$ 


\section{MHSalud}

URL www.una.ac.cr/mhsalud

Tabla 12. Resumen de promedios y desviaciones estándar de los puntajes obtenidos en el ítem 6

\begin{tabular}{|c|c|c|c|c|c|}
\hline & Grupo & Sexo & Promedio & Desviación estándar & $\mathbf{n}$ \\
\hline \multirow[t]{9}{*}{ Pre } & grupo control & mujer & 2,7143 & $\pm 0,7559$ & 7 \\
\hline & & hombre & 3,0000 & $\pm 0,0000$ & 7 \\
\hline & & Total & 2,8571 & $\pm 0,5345$ & 14 \\
\hline & grupo experimental & mujer & 2,8333 & $\pm 0,4082$ & 6 \\
\hline & & hombre & 2,1429 & $\pm 1,0690$ & 7 \\
\hline & & Total & 2,4615 & $\pm 0,8771$ & 13 \\
\hline & Total & mujer & 2,7692 & $\pm 0,5991$ & 13 \\
\hline & & hombre & 2,5714 & $\pm 0,8516$ & 14 \\
\hline & & Total & 2,6667 & $\pm 0,7338$ & 27 \\
\hline \multirow[t]{9}{*}{ Post } & grupo control & mujer & 2,7143 & $\pm 0,7559$ & 7 \\
\hline & & hombre & 2,4286 & $\pm 0,9759$ & 7 \\
\hline & & Total & 2,5714 & $\pm 0,8516$ & 14 \\
\hline & grupo experimental & mujer & 2,8333 & $\pm 0,4082$ & 6 \\
\hline & & hombre & 2,7143 & $\pm 0,7559$ & 7 \\
\hline & & Total & 2,7692 & $\pm 0,5991$ & 13 \\
\hline & Total & mujer & 2,7692 & $\pm 0,5991$ & 13 \\
\hline & & hombre & 2,5714 & $\pm 0,8516$ & 14 \\
\hline & & Total & 2,6667 & $\pm 0,7338$ & 27 \\
\hline
\end{tabular}

Tabla 13. Resumen de análisis de varianza de los puntajes obtenidos en el ítem 6 según sexo, grupo y medición

\begin{tabular}{cccc}
\hline Fuente & F & Sig. & Eta $^{2}$ \\
\hline MEDICION & 0,000 & 1,000 & 0,000 \\
MEDICION * GRUPO & 4,416 & $0,047^{*}$ & 0,161 \\
MEDICION * SEXO & 0,000 & 1,000 & 0,000 \\
MEDICION * GRUPO * SEXO & 4,416 & $0,047^{*}$ & 0,161 \\
GRUPO & 0,114 & 0,739 & 0,005 \\
SEXO & 0,673 & 0,420 & 0,028 \\
GRUPO * SEXO & 0,673 & 0,420 & 0,028 \\
\hline
\end{tabular}

$* \overline{\mathrm{p}}<0,05$

Tabla 14. Resumen de promedios y desviaciones estándar de los puntajes obtenidos en el ítem 7

\begin{tabular}{|c|c|c|c|c|c|}
\hline & Grupo & Sexo & Promedio & Desviación estándar & $\mathbf{n}$ \\
\hline \multirow[t]{9}{*}{ Pre } & grupo control & mujer & 1,5714 & $\pm 0,7868$ & 7 \\
\hline & & hombre & 1,2857 & $\pm 0,4880$ & 7 \\
\hline & & Total & 1,4286 & $\pm 0,6462$ & 14 \\
\hline & grupo experimental & mujer & 1,6667 & $\pm 0,8165$ & 6 \\
\hline & & hombre & 1,7143 & $\pm 0,4880$ & 7 \\
\hline & & Total & 1,6923 & $\pm 0,6304$ & 13 \\
\hline & Total & mujer & 1,6154 & $\pm 0,7679$ & 13 \\
\hline & & hombre & 1,5000 & $\pm 0,5189$ & 14 \\
\hline & & Total & 1,5556 & $\pm 0,6405$ & 27 \\
\hline \multirow[t]{9}{*}{ Post } & grupo control & mujer & 1,5714 & $\pm 0,7868$ & 7 \\
\hline & & hombre & 1,2857 & $\pm 0,4880$ & 7 \\
\hline & & Total & 1,4286 & $\pm 0,6462$ & 14 \\
\hline & grupo experimental & mujer & 2,8333 & $\pm 0,4082$ & 6 \\
\hline & & hombre & 2,7143 & $\pm 0,4880$ & 7 \\
\hline & & Total & 2,7692 & $\pm 0,4385$ & 13 \\
\hline & Total & mujer & 2,1538 & $\pm 0,8987$ & 13 \\
\hline & & hombre & 2,0000 & $\pm 0,8771$ & 14 \\
\hline & & Total & 2,0741 & $\pm 0,8738$ & 27 \\
\hline
\end{tabular}

Revista MHSalud® (ISSN: 1659-097X) Vol. 5. No. 2. Diciembre, 2008. 


\section{MHSalud}

URL www.una.ac.cr/mhsalud

Tabla 15. Resumen de análisis de varianza de los puntajes obtenidos en el ítem 7 según sexo, grupo y medición

\begin{tabular}{cccc}
\hline Fuente & F & Sig. & Eta $^{2}$ \\
\hline MEDICION & 26,545 & $0,000^{* * *}$ & 0,536 \\
MEDICION * GRUPO & 26,545 & $0,000^{* *}$ & 0,536 \\
MEDICION * SEXO & 0,157 & 0,696 & 0,007 \\
MEDICION * GRUPO * SEXO & 0,157 & 0,696 & 0,007 \\
GRUPO & 14,368 & $0,001 * *$ & 0,384 \\
SEXO & 0,575 & 0,456 & 0,024 \\
GRUPO * SEXO & 0,348 & 0,561 & 0,015 \\
& & & \\
\hline
\end{tabular}

$* * \mathrm{p}<0,01$

Tabla 16. Resumen de promedios y desviaciones estándar de los puntajes obtenidos en el ítem 8

\begin{tabular}{|c|c|c|c|c|c|}
\hline & Grupo & Sexo & Promedio & Desviación estándar & $\mathbf{n}$ \\
\hline \multirow[t]{9}{*}{ Pre } & grupo control & mujer & 2,8571 & $\pm 0,3780$ & 7 \\
\hline & & hombre & 2,8571 & $\pm 0,3780$ & 7 \\
\hline & & Total & 2,8571 & $\pm 0,3631$ & 14 \\
\hline & grupo experimental & mujer & 2,0000 & $\pm 0,8944$ & 6 \\
\hline & & hombre & 2,0000 & $\pm 0,8165$ & 7 \\
\hline & & Total & 2,0000 & $\pm 0,8165$ & 13 \\
\hline & Total & mujer & 2,4615 & $\pm 0,7763$ & 13 \\
\hline & & hombre & 2,4286 & $\pm 0,7559$ & 14 \\
\hline & & Total & 2,4444 & $\pm 0,7511$ & 27 \\
\hline \multirow[t]{9}{*}{ Post } & grupo control & mujer & 2,7143 & $\pm 0,4880$ & 7 \\
\hline & & hombre & 2,8571 & $\pm 0,3780$ & 7 \\
\hline & & Total & 2,7857 & $\pm 0,4258$ & 14 \\
\hline & grupo experimental & mujer & 2,8333 & $\pm 0,4082$ & 6 \\
\hline & & hombre & 2,8571 & $\pm 0,3780$ & 7 \\
\hline & & Total & 2,8462 & $\pm 0,3755$ & 13 \\
\hline & Total & mujer & 2,7692 & $\pm 0,4385$ & 13 \\
\hline & & hombre & 2,8571 & $\pm 0,3631$ & 14 \\
\hline & & Total & 2,8148 & $\pm 0,3958$ & 27 \\
\hline
\end{tabular}

Tabla 17. Resumen de análisis de varianza de los puntajes obtenidos en el ítem 8 según sexo, grupo y medición

\begin{tabular}{cccc}
\hline Fuente & F & Sig. & Eta $^{2}$ \\
\hline MEDICION & 8,774 & $0,007 * *$ & 0,276 \\
MEDICION * GRUPO & 12,313 & $0,002 * *$ & 0,349 \\
MEDICION * SEXO & 0,102 & 0,753 & 0,004 \\
MEDICION * GRUPO * SEXO & 0,052 & 0,822 & 0,002 \\
GRUPO & 5,841 & $0,024 *$ & 0,203 \\
SEXO & 0,064 & 0,803 & 0,003 \\
GRUPO * SEXO & 0,033 & 0,858 & 0,001 \\
$* \mathrm{p}<0,05 * * \mathrm{p}<0,01$ & &
\end{tabular}

Revista MHSalud® (ISSN: 1659-097X) Vol. 5. No. 2. Diciembre, 2008. 


\section{MHSalud}

URL www.una.ac.cr/mhsalud

Tabla 18. Resumen de promedios y desviaciones estándar de los puntajes obtenidos en el ítem 9

\begin{tabular}{|c|c|c|c|c|c|}
\hline & Grupo & Sexo & Promedio & Desviación estándar & $\mathbf{n}$ \\
\hline \multirow[t]{9}{*}{ Pre } & grupo control & mujer & 2,7143 & $\pm 0,7559$ & 7 \\
\hline & & hombre & 2,7143 & $\pm 0,7559$ & 7 \\
\hline & & Total & 2,7143 & $\pm 0,7263$ & 14 \\
\hline & grupo experimental & mujer & 2,0000 & $\pm 1,0954$ & 6 \\
\hline & & hombre & 2,2857 & $\pm 0,7559$ & 7 \\
\hline & & Total & 2,1538 & $\pm 0,8987$ & 13 \\
\hline & Total & mujer & 2,3846 & $\pm 0,9608$ & 13 \\
\hline & & hombre & 2,5000 & $\pm 0,7596$ & 14 \\
\hline & & Total & 2,4444 & $\pm 0,8473$ & 27 \\
\hline \multirow[t]{9}{*}{ Post } & grupo control & mujer & 2,0000 & $\pm 1,0000$ & 7 \\
\hline & & hombre & 2,1429 & $\pm 1,0690$ & 7 \\
\hline & & Total & 2,0714 & $\pm 0,9972$ & 14 \\
\hline & grupo experimental & mujer & 3,0000 & $\pm 0,0000$ & 6 \\
\hline & & hombre & 3,0000 & $\pm 0,0000$ & 7 \\
\hline & & Total & 3,0000 & $\pm 0,0000$ & 13 \\
\hline & Total & mujer & 2,4615 & $\pm 0,8771$ & 13 \\
\hline & & hombre & 2,5714 & $\pm 0,8516$ & 14 \\
\hline & & Total & 2,5185 & $\pm 0,8490$ & 27 \\
\hline
\end{tabular}

Tabla 19. Resumen de análisis de varianza de los puntajes obtenidos en el ítem 9 según sexo, grupo y medición

\begin{tabular}{cccc}
\hline Fuente & F & Sig. & Eta $^{2}$ \\
\hline MEDICION & 0,345 & 0,563 & 0,015 \\
MEDICION * GRUPO & 16,905 & $0,000^{* *}$ & 0,424 \\
MEDICION * SEXO & 0,038 & 0,846 & 0,002 \\
MEDICION * GRUPO * SEXO & 0,345 & 0,563 & 0,015 \\
GRUPO & 0,523 & 0,477 & 0,022 \\
SEXO & 0,188 & 0,668 & 0,008 \\
GRUPO * SEXO & 0,021 & 0,886 & 0,001 \\
\hline
\end{tabular}

$* * \mathrm{p}<0,01$

Tabla 20. Resumen de promedios y desviaciones estándar de los puntajes obtenidos en el ítem 10

\begin{tabular}{|c|c|c|c|c|c|}
\hline & Grupo & Sexo & Promedio & Desviación estándar & $\mathbf{n}$ \\
\hline \multirow[t]{9}{*}{ Pre } & grupo control & mujer & 1,5714 & $\pm 0,5345$ & 7 \\
\hline & & hombre & 1,8571 & $\pm 0,3780$ & 7 \\
\hline & & Total & 1,7143 & $\pm 0,4688$ & 14 \\
\hline & grupo experimental & mujer & 2,0000 & $\pm 0,6325$ & 6 \\
\hline & & hombre & 1,7143 & $\pm 0,4880$ & 7 \\
\hline & & Total & 1,8462 & $\pm 0,5547$ & 13 \\
\hline & Total & mujer & 1,7692 & $\pm 0,5991$ & 13 \\
\hline & & hombre & 1,7857 & $\pm 0,4258$ & 14 \\
\hline & & Total & 1,7778 & $\pm 0,5064$ & 27 \\
\hline \multirow[t]{9}{*}{ Post } & grupo control & mujer & 1,5714 & $\pm 0,5345$ & 7 \\
\hline & & hombre & 1,4286 & $\pm 0,5345$ & 7 \\
\hline & & Total & 1,5000 & $\pm 0,5189$ & 14 \\
\hline & grupo experimental & mujer & 2,1667 & $\pm 0,7528$ & 6 \\
\hline & & hombre & 2,4286 & $\pm 0,5345$ & 7 \\
\hline & & Total & 2,3077 & $\pm 0,6304$ & 13 \\
\hline & Total & mujer & 1,8462 & $\pm 0,6887$ & 13 \\
\hline & & hombre & 1,9286 & $\pm 0,7300$ & 14 \\
\hline & & Total & 1,8889 & $\pm 0,6980$ & 27 \\
\hline
\end{tabular}

Revista MHSalud® (ISSN: 1659-097X) Vol. 5. No. 2. Diciembre, 2008. 


\section{MHSalud}

URL www.una.ac.cr/mhsalud

Tabla 21. Resumen de análisis de varianza de los puntajes obtenidos en el ítem 10 según sexo, grupo y medición

\begin{tabular}{cccc}
\hline Fuente & F & Sig. & Eta $^{2}$ \\
\hline MEDICION & 0,793 & 0,383 & 0,033 \\
MEDICION * GRUPO & 6,642 & $0,017 *$ & 0,224 \\
MEDICION * SEXO & 0,055 & 0,817 & 0,002 \\
MEDICION * GRUPO * SEXO & 3,691 & 0,067 & 0,138 \\
GRUPO & 7,605 & $0,011^{*}$ & 0,248 \\
SEXO & 0,030 & 0,863 & 0,001 \\
GRUPO * SEXO & 0,060 & 0,809 & 0,003 \\
\hline
\end{tabular}

$* \mathrm{p}<0,05$

Tabla 22. Resumen de promedios y desviaciones estándar de los puntajes obtenidos en el ítem 11

\begin{tabular}{|c|c|c|c|c|c|}
\hline & Grupo & Sexo & Promedio & Desviación estándar & $\mathbf{n}$ \\
\hline \multirow[t]{9}{*}{ Pre } & grupo control & mujer & 2,1429 & $\pm 0,3780$ & 7 \\
\hline & & hombre & 1,8571 & $\pm 0,3780$ & 7 \\
\hline & & Total & 2,0000 & $\pm 0,3922$ & 14 \\
\hline & grupo experimental & mujer & 1,8333 & $\pm 0,4082$ & 6 \\
\hline & & hombre & 1,7143 & $\pm 0,4880$ & 7 \\
\hline & & Total & 1,7692 & $\pm 0,4385$ & 13 \\
\hline & Total & mujer & 2,0000 & $\pm 0,4082$ & 13 \\
\hline & & hombre & 1,7857 & $\pm 0,4258$ & 14 \\
\hline & & Total & 1,8889 & $\pm 0,4237$ & 27 \\
\hline \multirow[t]{9}{*}{ Post } & grupo control & mujer & 1,7143 & $\pm 0,7559$ & 7 \\
\hline & & hombre & 1,4286 & $\pm 0,5345$ & 7 \\
\hline & & Total & 1,5714 & $\pm 0,6462$ & 14 \\
\hline & grupo experimental & mujer & 2,6667 & $\pm 0,5164$ & 6 \\
\hline & & hombre & 2,8571 & $\pm 0,3780$ & 7 \\
\hline & & Total & 2,7692 & $\pm 0,4385$ & 13 \\
\hline & Total & mujer & 2,1538 & $\pm 0,8006$ & 13 \\
\hline & & hombre & 2,1429 & $\pm 0,8644$ & 14 \\
\hline & & Total & 2,1481 & $\pm 0,8182$ & 27 \\
\hline
\end{tabular}

Tabla 23. Resumen de análisis de varianza de los puntajes obtenidos en el ítem 11 según sexo, grupo y medición

\begin{tabular}{|c|c|c|c|}
\hline Fuente & $\mathbf{F}$ & Sig. & Eta $^{2}$ \\
\hline MEDICION & 5,306 & $0,031^{*}$ & 0,187 \\
\hline MEDICION * GRUPO & 34,016 & $0,000 * *$ & 0,597 \\
\hline MEDICION * SEXO & 0,406 & 0,530 & 0,017 \\
\hline MEDICION * GRUPO * SEXO & 0,406 & 0,530 & 0,017 \\
\hline GRUPO & 10,646 & $0,003 * *$ & 0,316 \\
\hline SEXO & 0,716 & 0,406 & 0,030 \\
\hline GRUPO * SEXO & 1,183 & 0,288 & 0,049 \\
\hline
\end{tabular}

$* \mathrm{p}<0,05 * * \mathrm{p}<0,01$ 


\section{MHSalud}

URL www.una.ac.cr/mhsalud

Tabla 24. Resumen de promedios y desviaciones estándar de los puntajes obtenidos en el ítem 12

\begin{tabular}{|c|c|c|c|c|c|}
\hline & Grupo & Sexo & Promedio & Desviación estándar & $\mathbf{n}$ \\
\hline \multirow[t]{9}{*}{ Pre } & grupo control & mujer & 2,1429 & $\pm 0,3780$ & 7 \\
\hline & & hombre & 1,8571 & $\pm 0,3780$ & 7 \\
\hline & & Total & 2,0000 & $\pm 0,3922$ & 14 \\
\hline & grupo experimental & mujer & 2,0000 & $\pm 0,6325$ & 6 \\
\hline & & hombre & 1,8571 & $\pm 0,8997$ & 7 \\
\hline & & Total & 1,9231 & $\pm 0,7596$ & 13 \\
\hline & Total & mujer & 2,0769 & $\pm 0,4935$ & 13 \\
\hline & & hombre & 1,8571 & $\pm 0,6630$ & 14 \\
\hline & & Total & 1,9630 & $\pm 0,5871$ & 27 \\
\hline \multirow[t]{9}{*}{ Post } & grupo control & mujer & 1,5714 & $\pm 0,7868$ & 7 \\
\hline & & hombre & 1,5714 & $\pm 0,5345$ & 7 \\
\hline & & Total & 1,5714 & $\pm 0,6462$ & 14 \\
\hline & grupo experimental & mujer & 2,3333 & $\pm 0,8165$ & 6 \\
\hline & & hombre & 2,7143 & $\pm 0,4880$ & 7 \\
\hline & & Total & 2,5385 & $\pm 0,6602$ & 13 \\
\hline & Total & mujer & 1,9231 & $\pm 0,8623$ & 13 \\
\hline & & hombre & 2,1429 & $\pm 0,7703$ & 14 \\
\hline & & Total & 2,0370 & $\pm 0,8077$ & 27 \\
\hline
\end{tabular}

Gráfico 1. Interacción de grupos y mediciones para el rendimiento en el ítem 2

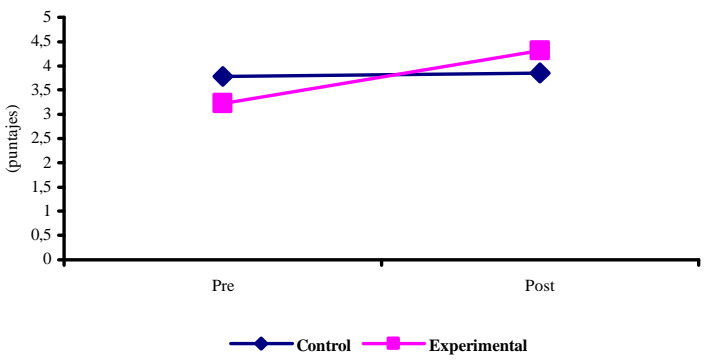

Gráfico 3. Interacción de grupos y mediciones para el rendimiento en el ítem 5

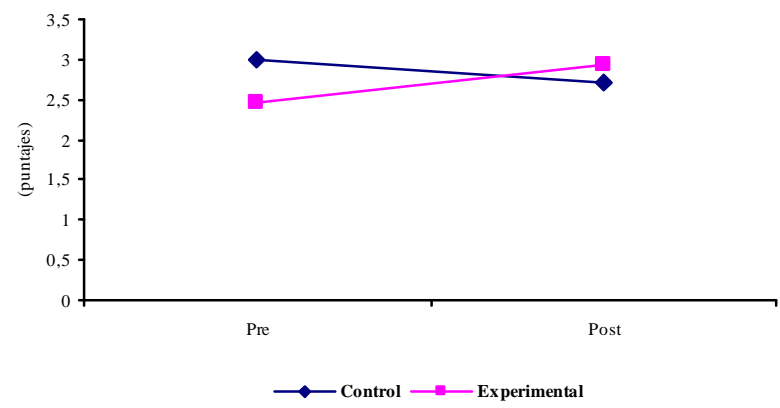

Gráfico 2. Interacción de grupos y mediciones para el rendimiento en el ítem 3

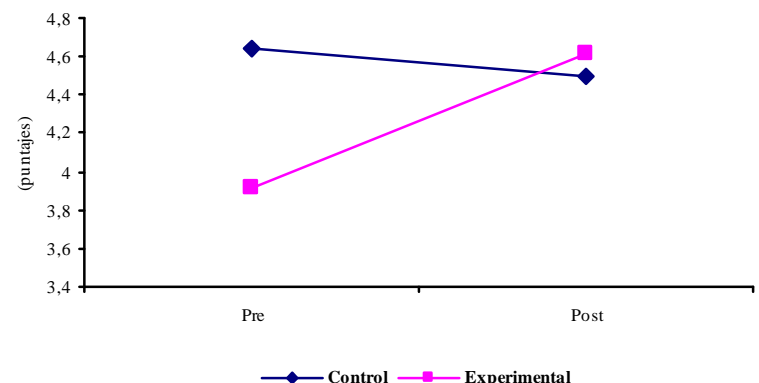

Gráfico 5. Interacción de grupos y mediciones para el rendimiento en el ítem 7

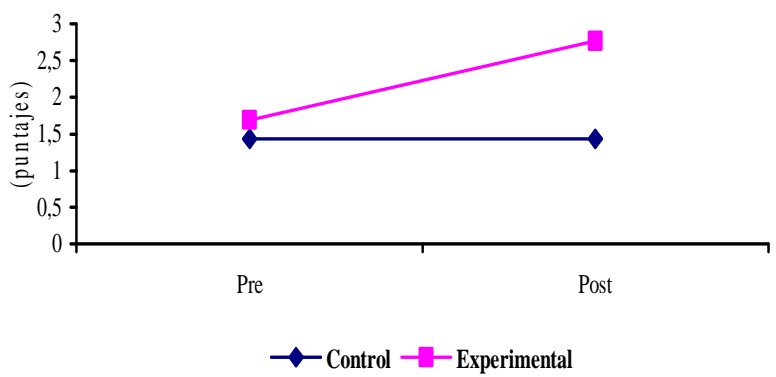

Revista MHSalud® (ISSN: 1659-097X) Vol. 5. No. 2. Diciembre, 2008. 
URL www.una.ac.cr/mhsalud

Gráfico 6. Interacción de grupos y mediciones para el rendimiento en el ítem 8

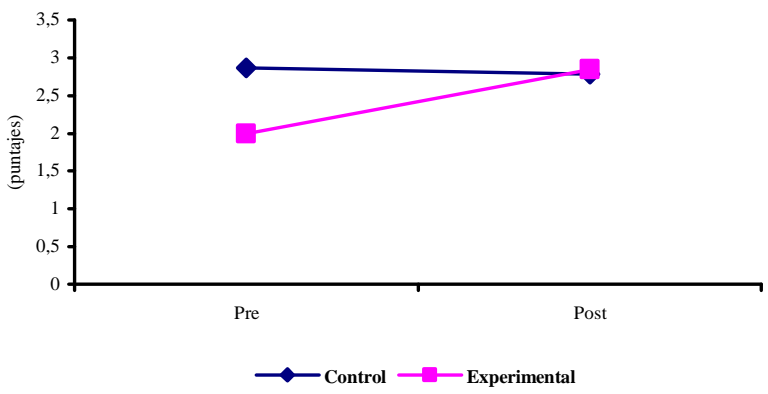

Gráfico 8. Interacción de grupos y mediciones para el rendimiento en el ítem 10

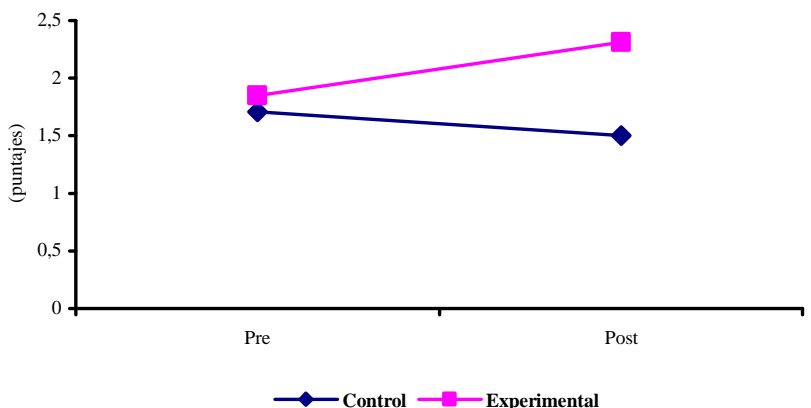

Gráfico 7. Interacción de grupos y mediciones para el rendimiento en el ítem 9

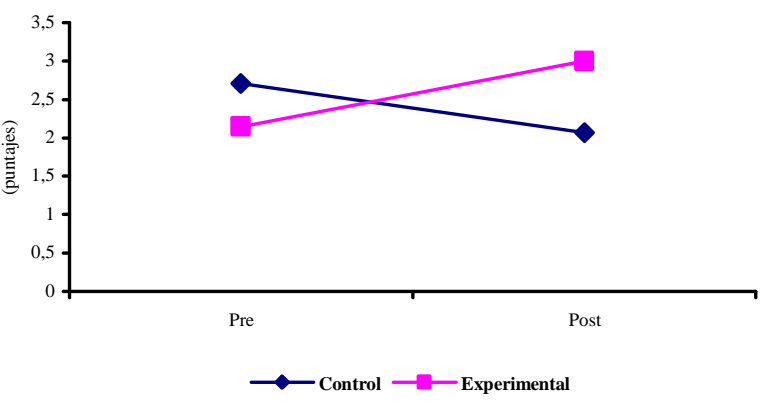

Gráfico 9. Interacción de grupos y mediciones para el rendimiento en el ítem 11

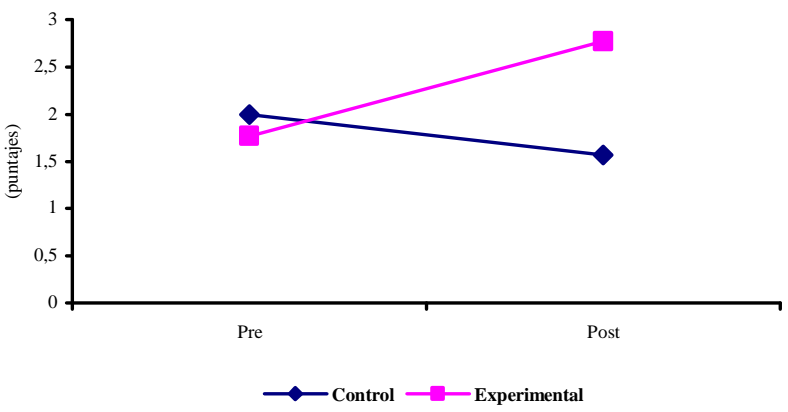

Tabla 25. Resumen de análisis de varianza de los puntajes obtenidos en el ítem 12 según sexo, grupo y medición

\begin{tabular}{cccc}
\hline Fuente & F & Sig. & Eta $^{2}$ \\
\hline MEDICION & & & 0,248 \\
MEDICION * GRUPO & 9,347 & $0,623 * *$ & 0,011 \\
MEDICION * SEXO & 1,461 & 0,239 & 0,289 \\
MEDICION * GRUPO * SEXO & 0,126 & 0,725 & 0,060 \\
GRUPO & 5,941 & $0,023 *$ & 0,205 \\
SEXO & 0,004 & 0,948 & 0,000 \\
GRUPO * SEXO & 0,525 & 0,476 & 0,022
\end{tabular}

$* \mathrm{p}<0,05 \quad * * \mathrm{p}<0,01$ 


\section{MHSalud}

URL www.una.ac.cr/mhsalud

Gráfico 4. Interacción de grupos, mediciones y sexo para el rendimiento en el ítem 6

Hombres

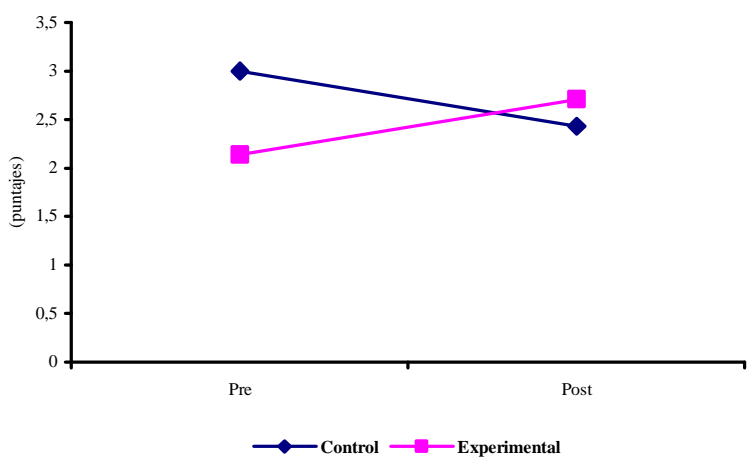

Mujeres

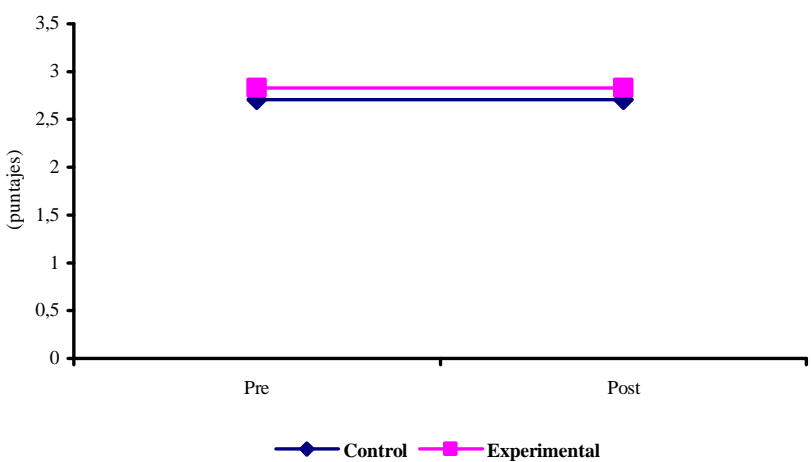

Gráfico 10. Interacción de grupos y mediciones para el rendimiento en el ítem 12

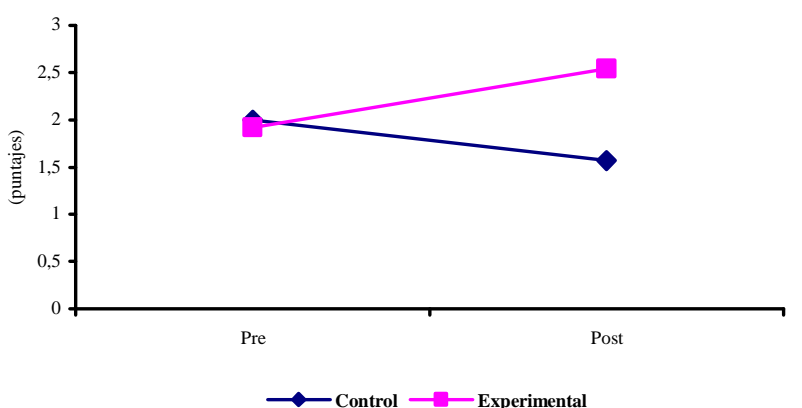

Se encontró efectos significativos y positivos del programa de actividades físico recreativas enfocados a las matemáticas sobre el rendimiento de los y las estudiantes en 10 de los 12 ítems que fueron aplicados para valorar el dominio de conceptos básicos de las matemáticas. Estos resultados se aprecian en los gráficos anteriores. Se encontró que los y las integrantes del grupo experimental mejoraron significativamente en todos los casos, mientras que el grupo control se mantenía estable o disminuía su rendimiento. Sólo en el ítem 6, se observó diferencias en el comportamiento de las mujeres y de los hombres, al mostrarse que el tratamiento fue solo efectivo para los hombres en este ítem. Existen diferencias iniciales en el desempeño del grupo control y experimental en los ítems 2, 3, 5, 8, 9 y 11 ; en todos los casos el grupo control tenía un mejor rendimiento en ese ítem, en comparación con el experimental, antes de iniciar el tratamiento (pre test). Esto se explica por haberse trabajado con grupos ya establecidos (diseño cuasiexperimental). 


\section{MHSalud}

URL www.una.ac.cr/mhsalud

\section{DISCUSIÓN}

En términos generales, el tratamiento aplicado fue positivo, lo cual va en la línea de otros estudios que han demostrado la efectividad del ejercicio y programas de actividad física para estimular el funcionamiento cognitivo (Gondola, 1987; Zervas, 1990; McMorris y Graydon, 2000, Gruber, 1986; Araya 2004; Aguilar, 1990; Moreira, 1993; Sanabria, 1995; Díaz, 1996; Alfaro y Salazar, 2001, Fisher, 1995; Serrano, Ramírez y Araya, en prensa; Rodríguez y Vega, 2004; González, Prado y Ramírez, 2004).

Los estudios metaanalíticos que se han publicado en el área de cognición y ejercicio con rango de edad amplio hasta personas mayores (Etnier et. al., 1997) así como los realizados con estudios efectuados en personas adultas mayores (Carazo, Araya y Salazar, 2007), coinciden en general, con el metaanálisis de Sibley y Etnier (2003), realizado con estudios de sujetos infantiles. El tener mayores niveles de actividad física estimula el funcionamiento cognitivo, desde la infancia hasta edades avanzadas. La presente investigación hace eco de esos hallazgos y los extiende al mostrar evidencias de esos efectos de la actividad física regular, en la edad preescolar en la cual no son frecuentes los estudios, como los autores anteriores indican y hace falta investigar más.

Zapata (1989), expresa que es de gran utilidad el uso de los juegos de aprestamiento para la lectura, la escritura y las matemáticas, ya que tienen la finalidad de iniciar al niño(a) en las actividades que lo conducirán al aprendizaje de estas disciplinas en el primer y segundo grado; a la vez, que prepararlo para la adquisición de estas habilidades dentro de un marco afectivo.

En contraparte, Amicale (1992) expresa que el movimiento participa en la elaboración y el desarrollo de todas las funciones mentales tales como la inteligencia, el lenguaje, la afectividad, conciencia, así como la interacción constante entre la parte mental y motora.

No obstante la formación recreativa es un instrumento en el desarrollo humano, cultural y depende fundamentalmente de los procesos educativos. La formación en contextos recreativos permite un alto nivel de eficacia en el aprendizaje gracias a que se realizan con una elevada motivación y un ambiente lúdico (Roque, 2001).

\section{CONCLUSIONES}

Al finalizar ésta investigación se pudo concluir que utilizar la educación física como un instrumento más para el aprendizaje de otras disciplinas, representa una excelente alternativa para los docentes de preescolar que buscan satisfacer las necesidades de aprendizaje de los niños y niñas que pronto irán a la escuela.

Se logró reforzar en los niños y las niñas los conocimientos básicos de las matemáticas por medio del movimiento, dado así que, el grupo experimental mostró mayores aciertos en comparación a la primera aplicación del cuestionario y mejoraron en comparación al grupo control.

El movimiento por medio de experiencias guiadas y planeadas, son parte indispensable en la vida de los niños y niñas, por lo tanto, el aprendizaje de materias académicas debe adaptarse a la necesidad del niño(a) de explorar y conocer su entorno.

El infante es un ser integral que necesita estimular y fortalecer a través de la exploración y la practica los conocimientos que va adquiriendo. 


\section{MHSalud}

URL www.una.ac.cr/mhsalud

El juego en el niño y niña es como el trabajo en el hombre, por lo tanto aprender jugando puede ser para ellos la mejor opción para su edad.

\section{REFERENCIAS}

Aguilar A., E.N. (1990). Estudio comparativo entre los resultados de aptitud física y los promedios en las asignaturas básicas del plan de estudios para determinar la importancia de la Educación Física en estudiantes de II ciclo de la Enseñanza General Básica, del circuito 02 de la Dirección Regional de Enseñanza de Turrialba. Tesis de graduación para optar al grado de Licenciado en Ciencias de la Educación con énfasis en Administración Educativa. Universidad de Costa Rica, Sede Regional del Atlántico, Facultad de Educación, Escuela de Administración Educativa, Costa Rica: Edgar Norberto Aguilar Araya.

Alfaro, Y. y Salazar, W. (2001). Efecto agudo del ejercicio físico en la inteligencia y la memoria en hombres, según la edad. Revista de ciencias del Ejercicio y la Salud, 1 (2): 1-11.

Amicale, E.P. (1992). El niño y la actividad física de 2 a 10 años. Barcelona, España: Paidotribo.

Araya, G.A. (2004). Educación a través del movimiento: un enfoque integral desde la educación física. Artículo no publicado.

Bolaño, M. y Thomas, E. (1994). Diccionario de recreación. Colombia: Talleres de publicación del politécnico colombiano Jaime Isaza Cadavid.

Bolaños, G. (1991). Educación por medio del movimiento y expresión corporal. San José, Costa Rica: Editorial EUNED.

Bustos, M.; Carrión, M.; García, J.; Guzmán, J.; Irigoyen A.; Larraya, I.; López, J.; Martínez, F.; Maseda, J.; Muriel, J.; Ruiz, M.; Sánchez, J.; Valle, R.; Velásquez, C. y Zoroza, A. (1999). Juegos populares. (1era. edición.). Madrid, España: Editorial Pila Teleña.

Carazo V., P.; Araya V., G. y Salazar R., W. (2007). Metaanálisis sobre el efecto del ejercicio en el funcionamiento cognitivo de adultos mayores. Revista Iberoamericana de Psicología del Ejercicio y el Deporte, 1 (2): 89-104

Céspedes R., E. (1987). Principios y técnicas recreativas para la expresión artística del niño. San José, Costa Rica: EUNED.

Cagigal, J.M. (1979). El deporte ante la educación. Boletín FIEP, Vol. 49, Número 48.

Decker, R. (1981). El deporte obligatorio en la escuela. Boletín Internacional de Educación Física. Vol. 51, Número 2, Junio.

Díaz Q., L.P. (1996). Efectos agudos del ejercicio en los procesos cognoscitivos en el niño. Tesis de grado presentada ante la Escuela de Educación Física y Deportes de la Universidad de Costa Rica para optar por el grado de Licenciada en Educación Física. San José, Costa Rica: Lilliam Patricia Díaz Quesada.

Etnier, J.; Salazar, W.; Landers, D.; Petruzello, S.S.; Han, M. y Novel, P. (1997). The influence of physical fitness and exercise upon cognitive functioning: A Meta - analysis. Journal of Sport and Exercise Psychology 19, 249-277. 


\section{MHSalud}

URL www.una.ac.cr/mhsalud

Fisher, E.P. (1995). The impact of play on development: A meta-analysis. Play \& Culture, 5 (2): 159181.

Gondola, J.C. (1987). The effects of a single bout of aerobic dancing on selected tests of creativity. Journal of Social Behavior and Personality, 2 : 275-278.

González, E.; Prado. E. y Ramírez M. (2004). Efectos de un programa de ejercicio aeróbico de baja intensidad en el estado anímico, cognitivo y físico en adultos mayores. Tesis Licenciatura en Ciencias del Deporte con énfasis en Salud. Escuela Ciencias del Deporte de la Universidad Nacional. Heredia, Costa Rica.

Gran libro de la maestra de preescolar. (2003). Evolución 5 años. Barcelona, España: Euromexico.

Gruber, J.P. (1986). Physical activity and self-steem development in children: A meta-analysis. En: Stull, G.A. \& Eckert, H.M. (eds.) Effects of physical activity on children. American Academy of Physical Education Papers, No.19, 30-48.

Huizinga, J. (1954). Homo Ludens. Madrid, España: editorial Alianza.

Lavega, P. (2000). Juegos y deportes populares tradicionales. España: INDE.

Lleixa, T. (1993). La Educación Física de 3 a 8 años. España: Editorial Paidotribo.

McMorris, T. y Graydon, J. (2000). The effect of incremental exercise on cognitive performance. International Journal of Sport Psychology, 31: 66-81.

Montes de Oca, F. y Romero, R. (2003). Acondicionamiento físico, funcionamiento cognitivo y estado anímico según el nivel de práctica de actividad física de hombres y mujeres adultos mayores costarricenses. Tesis Licenciatura en Ciencias del Deporte con énfasis en Salud. Escuela Ciencias del Deporte de la Universidad Nacional. Heredia, Costa Rica.

Moreira A., R. (1993). Efectos crónicos y agudos del ejercicio en los procesos cognitivos. Tesis de grado presentada ante la Escuela de Educación Física y Deportes de la Universidad de Costa Rica para optar al grado de Licenciado en Educación Física. San José, Costa Rica: Roberto Moreira Arguedas.

Morales, A. (1985). Cómo enseñar la matemática (1 edición). San José, Costa Rica: Serie Conchita, DECASA.

Moor, P. (1987). Die Bedeutung des Spieles in der Erziehung. [El juego en la educación] (4ta.edi.) Barcelona, España: Editorial Herder.

Rey, M. (2001). Maestra Jardinera. Los números van al jardín o el jardín va a los números. \# 57. Edición Mensual.

Rodríguez M. y Vega, J. (2004). Relación entre la edad, el nivel de práctica de actividad física y el funcionamiento cognitivo. Tesis Licenciatura en Ciencias del Deporte con énfasis en Salud. Escuela Ciencias del Deporte de la Universidad Nacional. Heredia, Costa Rica. 


\section{MHSalud}

URL www.una.ac.cr/mhsalud

Roque, R. (2001). Recreación formativa y políticas sociales. En: Ocio, tiempo libre y recreación. (1era edición). San José, Costa Rica: ICODER.

Rudik, P.A. (1986). Recreación para la vida. Puerto Rico: División Editorial.

Sanabria, I. (1995). Meta-análisis sobre los efectos del ejercicio en parámetros cognoscitivos. Tesis Licenciatura en Educación Física. Escuela de Educación Física y Deportes de la Universidad de Costa Rica. San José, Costa Rica.

Serrano, A., Ramírez, O. y Araya, G. (en prensa). Comparación del nivel cognitivo y de actividad física en niños (as) deportistas con edades de 6 a 13 años que asisten a la Escuela Deportiva Pedagógica (EDP-UNA) de la Escuela Ciencias del Deporte de la Universidad Nacional y niños (as) de la Escuela Centro Educativo Villalobos (ECEV) que no asisten a la EDP-UNA. Universidad Nacional, Heredia. Revista MHSalud.

Sibley, B.A. \& Etnier, J.L. (2003). The relationship between physical activity and cognition in children: A meta-analysis. Pediatric Exercise Science, 15, 243-256.

Tomporowsky, P.D. y Ellis, N.R. (1984). Effects of exercise on the physical fitness, intelligence and adaptative behavior of institutionalized mentally retarded adults. Applied Research in Mental Retardation 5:329-337.

Trigo, E. (1992). Juegos Motrices y creatividad (2da. ed.). Barcelona, España: Editorial Paidotribo.

Vaca, A. (1983). Los juegos en la Escuela Primaria. Bogotá, Colombia: Ministerio de Educación.

Villalobos, D. (2001). Actividad física como medios de prevención y tratamiento de la violencia intrafamiliar. En: Ocio, tiempo libre y recreación. (1era edición). San José, Costa Rica: ICODER

Woodburn, S. (1985). Desarrollo perceptual motor. Universidad Nacional. Heredia, Costa Rica: EUNA.

Zapata, O. (1989). Juego y aprendizaje escolar. México, D.F.: Editorial Pax México.

Zamora, R. (1988). Actividad física y salud. La Habana: Editorial Científico- Técnica, S.A.

Zervas, Y. (1990). Effect of a physical exercise session on verbal, visuospatial, and numerical ability. Perceptual and Motor Skills, $71: 379-383$.

Fecha de recepción: 29 de enero del 2008.

Fecha de aceptación: 05 de mayo del 2008.

Fecha de publicación: 31 de diciembre del 2008. 